\title{
Factores incidentes en el profesorado para la elección del aprendizaje invertido como referente metodológico
}

\author{
Santiago Pozo Sánchez \\ Jesús López Belmonte \\ Arturo Fuentes Cabrera \\ Juan Antonio López Núñez \\ Universidad de Granada. España. \\ santiagopozo@correo.ugr.es \\ jesuslopez@ugr.es \\ arturofuentes@ugr.es \\ juanlope@ugr.es
}

Recibido: 6/12/2019

Aceptado: 30/3/2020

Publicado: $3 / 7 / 2020$

\section{Resumen}

El objetivo de este estudio se centra en analizar los factores que inciden en el profesorado para utilizar o descartar el aprendizaje invertido (en inglés, flipped learning) como metodología, tales como el ámbito de la asignatura, la etapa educativa, el tipo de centro y el nivel de formación continua. La investigación se basa en un diseño no experimental y cuantitativo de tipo descriptivo y correlacional. Se ha empleado un cuestionario ad hoc validado como instrumento de medida sobre una muestra de 642 enseñantes de diversos centros docentes de España. Los resultados reflejan que el empleo del aprendizaje invertido como metodología didáctica es muy infrecuente, siendo las causas principales para ello el desconocimiento por parte del profesorado sobre cómo funciona la metodología y la falta de recursos técnico-pedagógicos existente en el centro donde imparte docencia. Su uso mayoritario se focaliza en las materias relacionadas con los ámbitos de la filología y las matemáticas, dentro de la educación secundaria y superior, y con especial protagonismo de los centros privados y concertados respecto a los públicos. Asimismo, la formación específica en aprendizaje invertido resulta ser un factor determinante para la aplicabilidad de dicho procedimiento, independientemente del nivel de conocimiento de otras metodologías activas.

Palabras clave: análisis de correlación; método activo; tecnologías de la información y de la comunicación; profesión docente 
Resum. Anàlisi correlacional de l'aplicabilitat de la classe inversa segons les particularitats de la pràctica docent

L'objectiu d'aquest estudi se centra a analitzar els factors que incideixen en el professorat per utilitzar o descartar la classe inversa (en anglès, flipped learning) com a metodologia, com ara l'àmbit de l'assignatura, l'etapa educativa, el tipus de centre i el nivell de formació contínua. La investigació es basa en un disseny no experimental i quantitatiu de tipus descriptiu i correlacional. S'hi ha emprat un quiestionari ad hoc validat com a instrument de mesura sobre una mostra de 642 docents de diversos centres educatius d'Espanya. Els resultats reflecteixen que la pràctica de la classe inversa com a metodologia didàctica és molt infreqüent. Les causes principals perquè succeeixi així són el desconeixement que pateix el professorat sobre com funciona la metodologia i la manca de recursos tecnicopedagògics existents al centre on imparteix docència. L'ús majoritari que se'n fa es focalitza en les matèries relacionades amb l'àmbit de la filologia i les matemàtiques, dins de l'educació secundària i superior, i amb especial protagonisme dels centres privats i concertats respecte als públics. Així mateix, la formació específica en classe invertida esdevé un factor determinant per a l'aplicabilitat d'aquest procediment, sense importar el nivell de coneixement d'altres metodologies actives.

Paraules clau: anàlisi de correlació; mètode actiu; tecnologies de la informació i de la comunicació; professió docent

\section{Abstract. Correlational analysis of the applicability of flipped learning according to the particularities of teaching practice}

This study analyses factors that influence teachers' decision to use or rule out flipped learning as a methodology; specifically, the scope of the subject, the educational stage, the type of institution and the level of continuing education. The research is based on a nonexperimental and quantitative descriptive and correlational design. A validated, ad hoc questionnaire was used as a measuring instrument in a sample of 642 teachers from various educational institutions in Spain. The results reflect that the use of flipped learning as a didactic methodology is very rare, mainly due to teachers' lack of knowledge about how the methodology works and the scarcity of techno-pedagogical resources where they teach. Flipped learning is primarily used for teaching topics related to language and mathematics in secondary and higher education, particularly in private and state-funded private schools with respect to public ones. Likewise, specific training in flipped learning was found to be a determining factor for the applicability of the methodology, regardless of the level of training in other active methodologies.

Keywords: correlation analysis; activity method; information and communication technologies; teaching profession

\section{Sumario}

$\begin{aligned} \text { 1. Introducción } & \text { 4. Discusión } \\ \text { 2. Método } & \text { 5. Conclusiones } \\ \text { 3. Resultados } & \text { Referencias bibliográficas }\end{aligned}$




\section{Introducción}

La mayor parte de las acciones educativas que se desarrollan hoy en día en las aulas no se adaptan a las particularidades del alumnado, por lo que se encuentran altamente descontextualizadas en relación con la práctica diaria de los discentes (Hinojo-Lucena et al., 2020; Hinojo-Lucena et al., 2018). La respuesta a esta desactualización de la acción docente se halla en la implementación de nuevas metodologías y tendencias pedagógicas para invertir las acciones, los espacios y los tiempos de los sistemas educativos tradicionales (Cabero y Barroso, 2018; Llanos y Bravo, 2017).

Una de estas alternativas pedagógicas actuales que puede dar respuesta al nuevo paradigma educativo es el aprendizaje invertido, metodología que constituye el objeto de estudio del presente trabajo. Este enfoque ha adquirido suma importancia en muy poco tiempo (Seery, 2015; Nikolopoulou, Akriotou y Gialamas, 2019; Zainuddin, Habiburrahim, Muluk y Keumala, 2019), puesto que se presenta como una excelente alternativa que aprovecha las potencialidades de las tecnologías de la información y la comunicación (TIC) en la práctica educativa, y se fundamenta en los principios de las metodologías activas (Froehlich, 2018; McLaughlin et al., 2014).

El aprendizaje invertido surge por el acertado trabajo de Jonathan Bergmann y Aaron Sams, expertos en materia educativa que durante el año 2012 elaboraron materiales en línea de carácter audiovisual destinados a aquellos alumnos que no podían asistir regularmente al aula, con la idea de que pudieran seguir el ritmo habitual de la clase (Bergmann y Sams, 2012). Desde aquel momento el aprendizaje invertido ha tenido una evolución muy notable y constante, contando con cada vez más seguidores en todo el mundo, atraídos por su efectividad en el desarrollo de los procesos docentes (He, Holton, Farkas y Warschauer, 2016).

El aprendizaje invertido (Sánchez, Sánchez y Ruiz, 2019) supone una completa inversión del sistema tradicional de aprendizaje (Bauer, Graney, Marshall y Sabieh, 2016). De esta manera, el periodo en el que el alumno está fuera del aula se dedica al trabajo específico con los contenidos de la asignatura (El Miedany, 2019; Khadri, 2016; Nouri, 2016; Zainuddin y Halili, 2016), empleando para ello diversas herramientas digitales, plataformas y cualquier elemento relacionado con las TIC que el docente haya elegido o confeccionado a tal efecto (Abeysekera y Dawson, 2015; Long, Cummins y Waugh, 2017; Schmidt y Ralph, 2016).

Concretamente, esta metodología innovadora centra el protagonismo absoluto de la acción educativa en el alumno, cambiando los roles tradicionales (Del Arco, Flores y Silva, 2016, 2019; Flores, Del Arco y Silva, 2016) y posibilitando que sea el discente quien decida y oriente su periodo de formación de manera autónoma (Ribosa, 2020; Salas y Lugo, 2019; Tourón y Santiago, 2015). Así este puede disponer de los contenidos didácticos en cualquier momento, siempre que disponga de un dispositivo móvil con acceso a Internet (Boelens, Voet y De Wever, 2018; López, Pozo y Pino, 2019). Todas estas 
particularidades de dicho enfoque provocan una interacción mayor entre todos los protagonistas del acto educativo que conviven en el aula (Castellanos, Sánchez y Calderero, 2017; Hwang, Lai y Wang, 2015). Supone, por tanto, un exponente de la enseñanza mixta que permite escoger lo mejor de la enseñanza en línea y de la enseñanza presencial (Lee, Lim y Kim, 2017; Mok, 2014), proporcionando un protagonismo al alumnado que le permite centrarse profusamente en los mecanismos de aprendizaje (Rué, 2007), en su autorregulación y en la adaptabilidad propia a sus periodos de mayor motivación y rendimiento (Báez y Clunie, 2019; Pereira, Fillol y Moura, 2019; Shih y Tsai, 2017; Tse, Choi y Tang, 2019).

A pesar de todo lo expuesto, el éxito de la implementación del aprendizaje invertido no es sencillo. Requiere de un alto grado de compromiso por parte de todos los agentes educativos, de formación específica, dedicación y esfuerzo por parte de los docentes (Chen y Summers, 2015), así como de un alto nivel de adquisición de las competencias digitales para poder generar los recursos en línea (Martín, Sáenz, Santiago y Chocarro, 2016; Padilla, Gámiz y Romero, 2020; Souto, Estévez, Iglesias y González, 2020) y saber administrar los espacios educativos digitales donde se desarrollan los contenidos teóricos (Gamboa y Sierra, 2017; López, Pozo, Fuentes y López, 2019). Todo ello precisa, además, de una alta cantidad de tiempo de dedicación para la generación continua de recursos que ayuden al alumno en su aprendizaje, tutorizar la formación del mismo y orientar de manera acertada su desarrollo académico (Jensen, Kummer y Godoy, 2015; Kwan y Foon, 2017).

Con respecto a su aplicabilidad, el aprendizaje invertido es extrapolable a cualquier etapa educativa (Calvillo, 2014), aunque resulta muy beneficiosa para aquellos que se encuentren más adelantados en sus conocimientos, pues es capaz de desarrollar de manera idónea los talentos más destacados (Tourón y Santiago, 2015). También es posible poner en práctica dicho enfoque en cualquier tipo de centro donde los recursos disponibles hagan posible su implantación, aunque no de manera exclusiva, debido a la posibilidad de aprovechar su aplicación en entornos de carácter abierto y flexible, formal e informal (Tucker, 2012).

\section{Método}

\subsection{Justificación y objetivos}

La era digital en la que nos encontramos inmersos hace que se torne altamente necesario que los docentes adapten sus metodologías pedagógicas al alumnado actual y a los nuevos espacios educativos (Area, Hernández y Sosa, 2016). Los discentes de hoy difieren sustancialmente de aquellos que copaban las aulas algunos años atrás. Se trata de un alumnado con altas dotes de lateralidad que, acostumbrado a realizar distintas tareas a la vez, es capaz de captar, procesar y utilizar información a altas velocidades (Pérez, 2018). Si esta capacidad potencialmente positiva no es aprovechada por parte del docente, puede conllevar 
a situaciones de aprendizaje ineficaz. En esta línea, Tourón y Santiago (2015) destacan un estudio realizado por la Universidad de Columbia (Estados Unidos) en el que se constató que el alumnado actual solo capta aproximadamente la mitad de las palabras del discurso del profesor, de forma que su capacidad de retentiva decrece exponencialmente conforme va pasando la hora lectiva.

Así pues, resulta necesario que el docente analice cuáles son las estrategias pedagógicas óptimas para que se produzcan aprendizajes significativos por parte de su alumnado y que aproveche al máximo el tiempo dedicado al proceso de adquisición de nuevos conocimientos. Bajo esta premisa el aprendizaje invertido se erige como un modelo pedagógico óptimo para contribuir a la educación del discente actual aprovechando al máximo sus beneficios (Mengual, López, Fuentes y Pozo, 2020).

A pesar de las enormes potencialidades que presenta dicho enfoque (Pozo, López, Moreno y López, 2019), las cifras de la difusión de su empleo entre el colectivo docente resultan insuficientes a priori. El profesor Alfredo Prieto Martín mantiene abierto en su blog (<http://profesor3punto0.blogspot.com>), desde el año 2016, un registro de docentes que se sirven del aula invertida (flippers) en España. Según los últimos datos recogidos del formulario, tan solo hay inscritos un total de 485 profesores, el 41,6\% de los cuales imparte docencia en educación superior (Prieto, 2019). A pesar de ello, es necesario tomar estas cifras con cautela, ya que el número de docentes que emplea el aula invertida y que no se encuentra inscrito en el registro puede ser significativo. Sin embargo, puede resultar ejemplificador de la tendencia ante la que nos encontramos aquellos profesores que empleamos dicho enfoque metodológico en nuestra labor diaria de forma exclusiva con respecto al resto de compañeros.

Por ende, surge la necesidad de indagar sobre aquellos factores que inciden a utilizarlo y conocer las causas que llevan al docente a emplearlo o desecharlo, de manera que la Administración educativa y los equipos directivos puedan considerar el grado de incidencia que ejercen dichos factores para solventar las deficiencias del profesorado y potenciar sus oportunidades y fortalezas.

En base a ello, el objetivo principal de este estudio se centra en analizar los factores que inciden en los docentes para utilizar o descartar el aprendizaje invertido como metodología dentro de su práctica educativa. De este enunciado general derivan los siguientes objetivos específicos que permitirán conducir todo el proceso investigador:

- Determinar la frecuencia de utilización del aprendizaje invertido del profesorado analizado durante la práctica docente.

- Conocer las motivaciones para rechazar el empleo del aprendizaje invertido como metodología.

- Concretar el ámbito de la materia que imparte el profesorado analizado, además de su etapa educativa y la tipología de su centro.

- Averiguar el nivel de formación continua del profesorado analizado.

- Analizar las asociaciones existentes entre la frecuencia de utilización del aprendizaje invertido y las particularidades de la práctica docente tales 
como la materia de impartición, la etapa educativa, la tipología del centro y el nivel de formación.

\subsection{Diseño de investigación y análisis de datos}

Esta investigación se ha efectuado desde un paradigma de investigación cuantitativa a través de un diseño de investigación no experimental de tipo descriptivo y correlacional. Todo ello teniendo en cuenta las consideraciones de expertos en el campo de la investigación como Hernández, Fernández y Baptista (2014).

El despliegue estadístico se ha efectuado con el programa Statistical Package for the Social Sciences (SPSS) en su versión 24. En él han intervenido aspectos básicos, como la media (M) y la desviación típica (DT), así como pruebas específicas para determinar la tendencia de la distribución, como el coeficiente de asimetría de Pearson (CAP) y el de apuntamiento de Fisher (CAF). Para conocer el grado de asociación entre las variables de estudio se ha empleado el test Chi-cuadrado de Pearson $\left(\chi^{2}\right)$, junto con la V de Cramer (V) y el coeficiente de contingencia (Cont) para concretar el grado de fuerza en la asociación. En todo el análisis estadístico se han tomado como diferencias estadísticamente significativas aquellos valores de $p<0,05$.

\subsection{Participantes}

En el estudio han participado un total de 642 docentes de diversos centros educativos de España. La selección de estos sujetos se ha llevado a cabo primeramente mediante una técnica de muestreo aleatorio tras la consulta en la base de datos que ofrece el Ministerio de Educación y Formación Profesional (<https://bit.ly/2TzPFiY>). A raíz de estos docentes se complementó el muestreo con la técnica de bola de nieve, a fin de expandir la cifra muestral a la máxima participación posible.

De estos sujetos, el $36,45 \%$ son hombres y el resto, mujeres, con una edad media de 43 años. En cuanto a la etapa educativa y al tipo de centro en el que imparte docencia la muestra analizada (figura 1), se observa una distribución desigual en la que predominan la educación primaria, con un 25,2\% $(n=162)$, y la educación secundaria obligatoria, con un 24,5\% $(n=157)$, como las etapas más numerosas, y la educación infantil, con un 13,6\% $(n=87)$, como la etapa educativa con menor número de docentes. En cuanto al tipo de centro en que el profesorado lleva a cabo su labor, el de los centros públicos supera ostensiblemente, con un $60,3 \%(n=445)$, al de los centros concertados, con un $16 \%(n=103)$, y privados, con un $14,7 \%(n=94)$, observándose una tendencia similar a la existente en el marco educativo nacional.

Con respecto a la materia o asignatura en la que imparte docencia el profesorado analizado, se observa una proporción equitativa entre los distintos ámbitos. Se ha optado por una agrupación de ese tipo para solventar las diferencias denominativas generadas por las desigualdades existentes en la etapa educativa. De esta forma, la diferencia de las frecuencias en la distribución de 
Figura 1. Etapa educativa del profesorado según tipos de centros

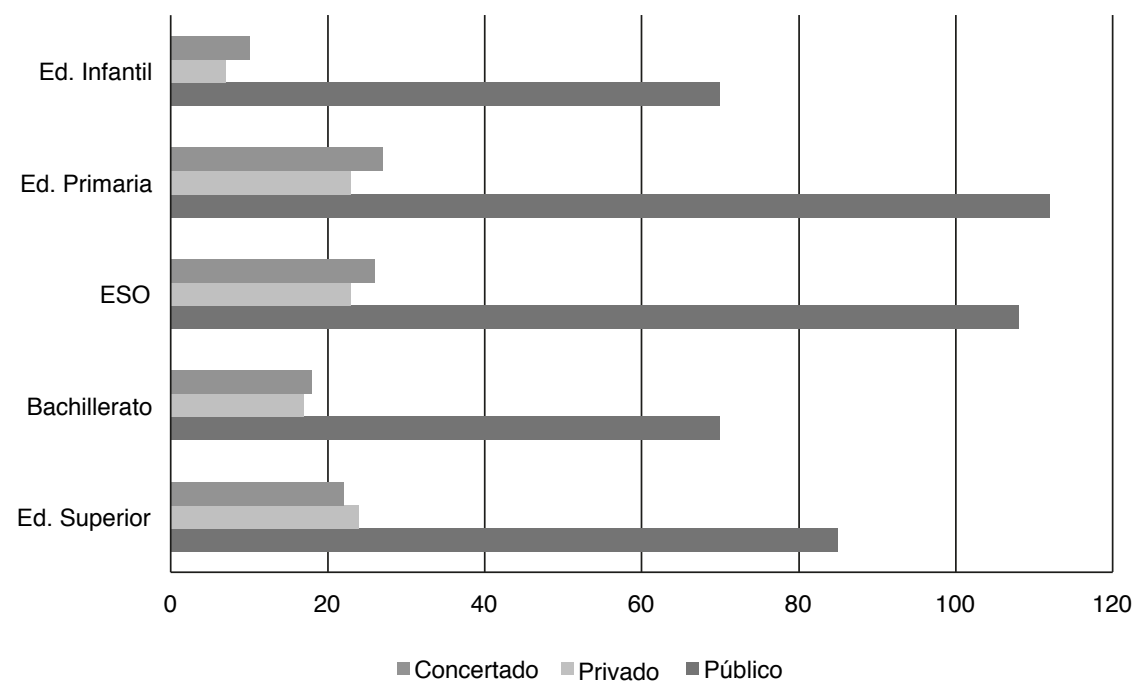

Fuente: elaboración propia.

la muestra es mínima entre el subgrupo muestral de mayor tamaño (ámbito de enseñanza de la lengua y la literatura: un $21,9 \% ; n=141$ ) y menor tamaño (ámbito de enseñanza de idiomas: un 17,8\%; $n=114$ ). Las frecuencias intermedias corresponden, en orden decreciente, al ámbito de enseñanza de las matemáticas (un 21,5\%; $n=138$ ), de las ciencias naturales (un 19,6\%; $n=126$ ) y de las ciencias sociales (un 19,2\%; $n=123$ ).

\subsection{Instrumento}

Un cuestionario de tipo ad hoc ha sido el instrumento escogido para recoger los datos necesarios para dar respuesta a los objetivos planteados. El instrumento en cuestión ha sido confeccionado en base a otras herramientas validadas reportadas de la literatura científica (Driscoll, 2012; Martín et al., 2016; Santiago y Bergmann, 2018). El cuestionario se articula en 42 ítems que se encuentran clasificados en tres dimensiones (socioeducativa, usabilidad, formativa). En su mayoría, las cuestiones se encuentran configuradas siguiendo un formato de respuesta en una escala de Likert de 4 puntos, siendo 1 el valor más negativo y 4, el más positivo.

Este instrumento ha sido sometido a un doble proceso de validación. En primer lugar, se realizó una validación cualitativa a través de un método Delphi. En él intervinieron un total de 12 expertos en metodologías activas de distintas universidades, los cuales otorgaron una opinión favorable $(\mathrm{M}=5,02$; $\mathrm{DT}=0,31$; mín. = 1; máx. =6). A su vez, estos especialistas ofrecieron retroa- 
limentaciones para optimizar el cuestionario que se centraron en la mejora de la redacción y la reducción del número de ítems. Todo enfocado hacia la optimización de la interpretación y hacia la eficaz cumplimentación del mismo. Seguidamente, se aplicaron pruebas como Kappa de Fleiss y W de Kendall en los juicios emitidos por los jueces para determinar la concordancia y la pertinencia de las observaciones, siendo los resultados bastante adecuados $(\mathrm{K}=0,84 ; \mathrm{W}=0,87)$.

La validación cuantitativa se realizó por medio de un análisis factorial exploratorio siguiendo el método de componentes principales con rotación varimax. Las pruebas efectuadas reflejaron dependencia entre las variables establecidas (prueba de esfericidad de Bartlett $=2837.41 ; p<0,001$ ) y una adecuación muestral pertinente (test de Kaiser-Meyer-Olkin =0,88).

En última instancia, se analizó la fiabilidad del cuestionario mediante diversos procedimientos estadísticos como el alfa $(\alpha)$ de Cronbach $(0,85)$, la fiabilidad compuesta $(0,86)$ y la varianza media extractada $(0,83)$.

\subsection{Variables de estudio}

Con base en la estructuración dimensional del instrumento, se han formulado las siguientes variables de estudio:

- Frecuencia de utilización del aprendizaje invertido durante la práctica docente.

- Motivaciones para rechazar el empleo del aprendizaje invertido como metodología.

- Concreción del ámbito según la materia en el que se imparte docencia.

- Etapa educativa del profesorado analizado.

- Tipo de centro en el que imparte docencia el profesorado analizado.

- Tipo de formación continua del profesorado analizado.

\subsection{Procedimiento}

La investigación dio comienzo en diciembre de 2018 con la primera versión del cuestionario, la cual fue mejorada y optimizada en base a las consideraciones de los expertos tras el proceso de evaluación anteriormente mencionado. Una vez diseñado y validado el instrumento, se envió telemáticamente a los distintos participantes que fueron seleccionados en un primer momento. Seguidamente, el cuestionario fue enviado a otros profesionales de la educación, estableciendo una cadena de envíos a partir de los primeros participantes. La fase de cumplimentación del cuestionario se prolongó durante 3 meses. Durante este tiempo los investigadores se encontraron activos para resolver las dudas y las inquietudes de los participantes. Una vez transcurrido este periodo temporal, se procedió a la preparación de los datos y a la importación al programa estadístico para efectuar todo el análisis pertinente que condujera al alcance de los objetivos marcados. 


\section{Resultados}

Iniciando el estudio de los resultados con el análisis de la frecuencia de utilización del aprendizaje invertido durante la práctica docente (figura 2), se ha obtenido que el 79,3\% $(n=509)$ de docentes analizados no emplea dicha metodología frente al 20,7\% $(n=133)$ que sí la emplea, independientemente del nivel de frecuencia. Dentro del subgrupo muestral que decide utilizar el aprendizaje invertido destacan por encima del resto aquellos que lo utilizan con una alta frecuencia (un 12\%; $n=77$ ), mostrando resultados similares los que lo emplean con cierta frecuencia (un $5 \% ; n=32$ ) y solo en algunos casos (un $3,7 \% ; n=24$ ).

Figura 2. Frecuencia de utilización del aprendizaje invertido por parte de los docentes

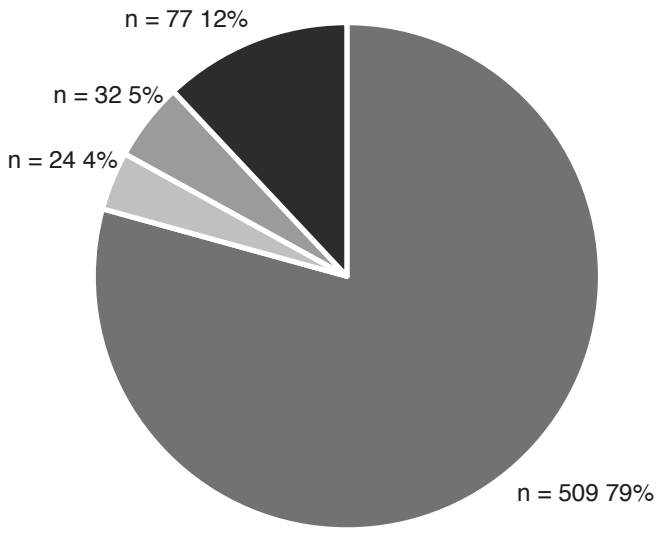

Nunca: no utiliza dicha metodología

Baja: la utiliza solo en algunos casos y con poca asiduidad

Media: la utiliza con cierta frecuencia y de manera continuada

Alta: la utiliza en prácticamente la totalidad de casos

Fuente: elaboración propia.

De esta forma, analizando las motivaciones para rechazar el empleo del aprendizaje invertido como metodología (tabla 1), observamos que este subgrupo muestral (un 79,3\%; $n=509$ ) alude como principales causas el desconocimiento sobre cómo funciona la metodología del aprendizaje invertido (un $38,7 \% ; n=197)$ y la falta de recursos técnico-pedagógicos en el centro donde imparte docencia (un 26,7\%;n=136). Como motivaciones alternativas y menos frecuentes destacan la falta de adecuación a las necesidades de su alumnado (un $13,5 \% ; n=69$ ), la ausencia de formación docente para abordar la metodología (un $12,4 \% ; n=63$ ) y la aprensión hacia el empleo de recursos tecnológicos en el aula (un $8,7 \% ; n=44$ ).

Abordando el tipo de formación continua de los docentes (tabla 1), se ha obtenido que mayoritariamente los participantes aluden al hecho de tener una formación insuficiente en aprendizaje invertido, a pesar de sí estar debidamente formados en otras metodologías emergentes (un 44,1\%; $n=283$ ), resultados seguidos de un importante número de docentes que afirma tener suficiente 
Tabla 1. Motivaciones para rechazar el aprendizaje invertido y tipo de formación del profesorado analizado

\begin{tabular}{lc}
\hline Motivaciones para rechazar el empleo del aprendizaje invertido* & $\boldsymbol{n}(\%)$ \\
\hline Por desconocer el funcionamiento de la metodología & $197(38,7)$ \\
\hline Por falta de formación para poder llevarla a cabo & $63(12,4)$ \\
\hline Por falta de recursos tecnológicos en el centro & $136(26,7)$ \\
\hline Por considerar que no contribuye de forma efectiva al aprendizaje discente & $69(13,5)$ \\
\hline Por presentar aprensión hacia el uso de la tecnología en el aula & $44(8,7)$ \\
\hline Tipo de formación continua del profesorado analizado** & $\boldsymbol{n}(\%)$ \\
\hline Suficiente en metodologías activas y en aprendizaje invertido & $252(39,2)$ \\
\hline Suficiente en metodologías activas pero insuficiente en aprendizaje invertido & $283(44,1)$ \\
\hline Insuficiente en metodologías activas pero suficiente en aprendizaje invertido & $32(5)$ \\
\hline Insuficiente en metodologías activas y en aprendizaje invertido & $75(11,7)$ \\
\hline
\end{tabular}

* M: 1,978; DT: 1,242; Curtosis: $-1,219 ;{ }^{* *}$ M: 1,89; DT: 0,984; Curtosis: 0,268

Fuente: elaboración propia.

formación en ambos casos (un 39,2\%; $n=252$ ). Solo el 11,7\% de la muestra afirma tener formación insuficiente en general $(n=75)$ y aun en menor número alude poseer suficiente formación específica en aprendizaje invertido, pero insuficiente en otras metodologías emergentes (un 5\%; $n=32$ ).

Por último, se abordan los resultados arrojados en el grado de utilización del aprendizaje invertido, y por lo que se refiere a las variables relacionadas con las particularidades de la práctica docente (tabla 2), se ha obtenido significancia estadística en cada una de ellas.

Con respecto al ámbito de aplicación, los docentes que imparten materias relacionadas con la filología o con las matemáticas son más propensos a emplear el aprendizaje invertido en su labor instructiva, aunque esta correlación presenta una fuerza de asociación media-débil $(\mathrm{V}=0,2$; Cont $=0,35)$.

En cuanto a la etapa educativa, la frecuencia de utilización del aprendizaje invertido es mucho más alta en ESO, bachillerato y educación superior que en educación primaria y educación infantil, donde es prácticamente nula. En este caso, la fuerza de asociación de la correlación es media-alta $(V=0,35$; Cont $=0,64)$.

En lo referente al tipo de centro, se observa una tendencia mayoritaria de los que son concertados y privados de emplear con mayor frecuencia el aprendizaje invertido con respecto a los centros públicos, aunque esta correlación presenta una fuerza de asociación media-débil $(\mathrm{V}=0,23$; Cont $=0,34)$.

Por último, aquellos docentes que presentan suficiente formación tanto en aprendizaje invertido como en metodologías activas tienen una propensión mucho más alta a emplear dicha metodología, habiéndose obtenido una fuerza de asociación muy alta en dicha correlación $(\mathrm{V}=0,51$; Cont $=0,103)$. Los docentes con formación parcial materializada en una suficiente formación en aprendizaje invertido pero insuficiente en metodologías activas presentan frecuencias relativas más altas que los dos grupos restantes, pero aun así muestran una reducida asiduidad al empleo del aprendizaje invertido. 
Tabla 2. Asociación estadística entre la frecuencia de utilización del aprendizaje invertido y las variables relacionadas con las particularidades de la práctica docente

\begin{tabular}{|c|c|c|c|c|c|c|c|}
\hline \multirow[b]{2}{*}{ Likert } & \multicolumn{4}{|c|}{$\begin{array}{c}\text { Frecuencia de uso del aprendizaje } \\
\text { invertido } n(\%)\end{array}$} & \multicolumn{3}{|c|}{ Parámetros } \\
\hline & Nulo & Bajo & Medio & Alto & $x^{2}(g l)$ & $p$-valor & V (Cont) \\
\hline Ámbito & & & & & $26,2(12)$ & 0,009 & $0,198(0,35)$ \\
\hline Filología & $106(16,5)$ & $7(1,1)$ & $12(1,9)$ & $16(2,5)$ & & & \\
\hline Matemáticas & $108(16,8)$ & $1(0,2)$ & $5(0,8)$ & $24(3,7)$ & & & \\
\hline Idiomas & $101(15,7)$ & $3(0,5)$ & $3(0,5)$ & $7(1,1)$ & & & \\
\hline C. sociales & $88(13,7)$ & $7(1,1)$ & $7(1,1)$ & $21(3,3)$ & & & \\
\hline C. naturales & $106(16,5)$ & $6(0,9)$ & $5(0,8)$ & $9(1,4)$ & & & \\
\hline Etapa & & & & & $88,4(12)$ & $<0,001$ & $0,348(0,64)$ \\
\hline Infantil & $87(13,6)$ & $-(0)$ & $-(0)$ & $-(0)$ & & & \\
\hline Primaria & $154(24)$ & $6(0,9)$ & $2(0,3)$ & $-(0)$ & & & \\
\hline ESO & $110(17,1)$ & $4(0,6)$ & $14(2,2)$ & $29(4,5)$ & & & \\
\hline Bachillerato & $68(10,6)$ & $6(0,9)$ & $8(1,2)$ & $23(3,6)$ & & & \\
\hline Superior & $89(13,9)$ & $8(1,2)$ & $9(1,4)$ & $25(3,9)$ & & & \\
\hline Tipo de centro & & & & & $36,5(6)$ & $<0,001$ & $0,232(0,34)$ \\
\hline Público & $374(58,3)$ & $20(3,1)$ & $19(3)$ & $32(5)$ & & & \\
\hline Privado & $65(10,1)$ & $1(0,2)$ & $7(1,1)$ & $21(3,3)$ & & & \\
\hline Concertado & $70(10,9)$ & $3(0,5)$ & $6(0,9)$ & $24(3,7)$ & & & \\
\hline Formación & & & & & 225,5 (9) & $<0,001$ & $0,510(1,03)$ \\
\hline Suficiente & $132(20,6)$ & $14(2,2)$ & $30(4,7)$ & $76(11,8)$ & & & \\
\hline Insuficiente & $75(11,7)$ & $-(0)$ & $-(0)$ & $-(0)$ & & & \\
\hline Parcial-NoME & $23(3,6)$ & $6(0,9)$ & $2(0,3)$ & $1(0,2)$ & & & \\
\hline Parcial-NoFL & $279(43,5)$ & $4(0,6)$ & $-(0)$ & $-(0)$ & & & \\
\hline
\end{tabular}

Fuente: elaboración propia.

\section{Discusión}

El presente estudio ha analizado los factores que inciden en el profesorado para utilizar o descartar el aprendizaje invertido como metodología dentro de su práctica docente. De esta forma, se ha estudiado la frecuencia con que se sirve de él. Los resultados nos muestran que solo dos de cada diez enseñantes emplean dicha metodología y solo uno de cada diez lo hace frecuentemente. A pesar de que el aprendizaje invertido se erige como una alternativa pedagógica que puede dar respuesta al nuevo paradigma educativo, las frecuencias de empleo en la muestra de estudio son muy reducidas en la práctica, hecho que contrasta con sus grandes potencialidades como alternativa metodológica (Froehlich, 2018; McLaughlin et al., 2014) y con la supuesta importancia que ha adquirido en la última década (Seery, 2015; Zainuddin et al., 2019). 
Tras el análisis sobre las causas y las motivaciones para rechazar el empleo del aprendizaje invertido como metodología, la mayor parte de los docentes ha aludido al desconocimiento sobre cómo funciona y a la falta de recursos técnico-pedagógicos en el centro donde imparte docencia. Como motivaciones alternativas y menos frecuentes destacan la falta de adecuación a las necesidades de su alumnado, la ausencia de formación docente para abordar la metodología y la aprensión hacia el empleo de recursos tecnológicos en el aula. Estas causas pueden imbricarse en el ya mencionado alto grado de compromiso docente (Chen y Summers, 2015), en el esfuerzo necesario para preparar los espacios educativos digitales donde se desarrolla la metodología (Gamboa y Sierra, 2017; López, Pozo y Pino, 2019) y en el coste temporal que conlleva la tutorización y la orientación del aprendizaje activo (Jensen et al., 2015; Kwan y Foon, 2017).

Por otra parte, en el análisis de las asociaciones existentes entre la frecuencia de utilización del aprendizaje invertido y las particularidades de la práctica docente se ha podido comprobar que, efectivamente, dichas particularidades afectan a la asiduidad del empleo de dicha metodología. En primer lugar, la frecuencia de utilización del aprendizaje invertido ha resultado ser mucho mayor en ESO, bachillerato y educación superior que en educación primaria e infantil, donde es prácticamente nula. Los docentes que imparten materias del ámbito de la filología o las matemáticas son más propensos a emplear el aprendizaje invertido en su práctica diaria. Asimismo, se ha observado una tendencia mayoritaria de los centros concertados y privados de emplear con mayor frecuencia el aprendizaje invertido con respecto a los centros públicos. En la línea expuesta por otros estudios se observa un mayor protagonismo de esta metodología en etapas educativas superiores, en particular en la educación secundaria y el ámbito universitario (Calvillo, 2014), especialmente en aquellas en las que las destrezas del alumnado en competencia digital sean superiores (Tourón y Santiago, 2015). Existe, además, una clara tendencia de mayor utilización del aprendizaje invertido en aquellos centros debidamente dotados de recursos tecnológicos que hagan posible su puesta en práctica (Tucker, 2012), hecho que puede explicar los resultados más positivos en los centros privados y concertados.

Asimismo, aquellos enseñantes que presentan suficiente formación tanto en aprendizaje invertido como en metodologías activas muestran una propensión mucho más alta a emplear dicha metodología, constatación que se encuentra en consonancia con otros estudios que subrayan la importancia de la formación y el compromiso docente para ponerla en práctica (Chen y Summers, 2015; Jensen et al., 2015; Kwan y Foon, 2017), así como un adecuado nivel de competencia digital para poder generar los recursos audiovisuales en línea (Gamboa y Sierra, 2017; López, Pozo y Pino, 2019; Martín et al., 2016). 


\section{Conclusiones}

El empleo del aprendizaje invertido como metodología didáctica es muy infrecuente. Las causas principales para ello son el desconocimiento sobre cómo funciona la metodología por parte del profesorado y la falta de recursos técnico-pedagógicos en el centro donde trabajan. En lo referente a la incidencia de la asignatura impartida por el docente, su uso mayoritario se focaliza en las materias relacionadas con el ámbito de la filología y las matemáticas. En lo concerniente a la etapa educativa y al tipo de centro, el aprendizaje invertido cuenta con un uso mayoritario dentro de la educación secundaria y superior, con especial protagonismo de los centros privados y concertados respecto a los públicos. Asimismo, la formación específica del profesorado en aprendizaje invertido resulta ser un factor determinante para la aplicabilidad de dicho procedimiento, de manera que son los docentes con un nivel más elevado de formación sobre metodologías activas, en general, y sobre aprendizaje invertido, en particular, los que ser sirven de él con mayor frecuencia.

Las principales limitaciones del estudio se centran en dos cuestiones principales relacionadas con los hallazgos de la literatura científica y con las características de la muestra de análisis. Por un lado, son escasos los estudios hallados en la literatura científica que traten específicamente los factores incidentes en la elección del profesorado para utilizar o descartar el aprendizaje invertido durante su práctica profesional, de manera que se torna altamente compleja la discusión de los resultados aquí obtenidos. Por otro lado, el reducido tamaño del subgrupo muestral que sí emplea el aprendizaje invertido como metodología habitual provoca una repartición desigual de las frecuencias dentro de cada subgrupo muestral. De esta forma, a partir de los resultados obtenidos en la presente investigación, se fomenta la necesidad de llevar a cabo análisis concretos sobre los factores incidentes en la aplicabilidad de las metodologías didácticas, de manera que la Administración educativa y los equipos directivos puedan considerar el grado de incidencia que ejercen dichos factores para solventar las deficiencias del profesorado y potenciar sus oportunidades y fortalezas.

Siguiendo esta línea y teniendo en cuenta las motivaciones reseñadas por el profesorado analizado para prescindir del aprendizaje invertido (escasez de recursos y/o de formación), como futura línea de investigación se propone la realización de un estudio de similares características, dejando un margen temporal específico para analizar los efectos del tiempo en lo referente al posible incremento de la dotación de recursos tecnológicos en las aulas, a la reposición de jóvenes enseñantes con una formación más amplia en TIC y metodologías emergentes y a la mayor difusión del aprendizaje invertido entre el colectivo docente como metodología pertinente para la acción formativa. 


\section{Referencias bibliográficas}

Abeysekera, L. y Dawson, P. (2015). Motivation and cognitive load in the flipped classroom: Definition, rationale and a call for research. Higher Education Research \& Development, 34(1), 1-26. <https://doi.org/10.1080/07294360.2014.934336>

Arco, I. del; Flores, O. y SiLva, P. (2016). Flipped classroom en las aulas universitarias: El caso de la formación de maestros y psicopedagogos. En C. González (presidencia). XVI Congreso Nacional y VII Congreso Iberoamericano de Pedagogía. Madrid (España), del 28 al 30 de junio de 2016. Recuperado de <https://cutt.ly/StmTrch>.

Arco Bravo, I. del; Flores, Ó. y Silva, P. (2019). El desarrollo del modelo flipped classroom en la universidad: Impacto de su implementación desde la voz del estudiantado. Revista de Investigación Educativa, 37(2), 451-469. <https://doi.org/10.6018/rie.37.2.327831>

Area, M.; Hernández, V. y Sosa, J.J. (2016). Modelos de integración didáctica de las TIC en el aula. Comunicar: Revista Cientifica Iberoamericana de Comunicación y Educación, 24(47), 79-87. <https://doi.org/10.3916/C47-2016-08>

Báez, C.I. y Clunie, C.E. (2019). Una mirada a la Educación Ubicua. RIED: Revista Iberoamericana de Educación a Distancia, 22(1), 325-344. <https://doi.org/10.5944/ried.22.1.22422>

Bauer, C.; Graney, J.M.; Marshall, H.W. y Sabieh, C. (2016). Flipped learning in TESOL: Definitions, approaches, and implementation. Tesol Journal, 7(2), 429-437. <https://doi.org/10.1002/tesj.250>

Bergmann, J. y Sams, A. (2012). Flip Your Classroom: Reach every student in every class every day. Washington DC: ISTE.

Boelens, R.; Voet, M. y De Wever, B. (2018). The design of blended learning in response to student diversity in higher education: Instructors' views and use of differentiated instruction in blended learning. Computers \& Education, 120, 197 212 . <https://doi.org/10.1016/j.compedu.2018.02.009>

Bognar, B.; Sablić, M. y ŠKugor, A. (2019). Flipped learning and Online Discussion in Higher Education Teaching. En C. Reidsema, L. Kavanagh, R. Hadgraft y N. Smith (eds.). The flipped classroom: Practice and practices in higher education (pp. 371-392). Nueva York: Springer. <https://doi.org/10.1007/978-3-030-01551-0_19>

Cabero, J. y Barroso, J. (2018). Los escenarios tecnológicos en Realidad Aumentada (RA): Posibilidades educativas en estudios universitarios. Aula Abierta, 47(3), 327-336. <https://doi.org/10.17811/rifie.47.3.2018.327-336>

Calvillo, A.J. (2014). El modelo flipped learning aplicado a la materia de música en el cuarto curso de educación secundaria obligatoria: Una investigación-acción para la mejora de la práctica docente y del rendimiento académico del alumnado. Tesis doctoral. Universidad de Valladolid, España.

Castellanos, A.; Sánchez, C. y Calderero, J.F. (2017). Nuevos modelos tecnopedagógicos: Competencia digital de los alumnos universitarios. Revista Electrónica de Investigación Educativa, 19(1), 1-9.

<https://doi.org/10.24320/redie.2017.19.1.1148> 
Chen, H.L. y Summers, K.L. (2015). Developing, using, and interacting in the flipped learning movement: Gaps among subject areas. The International Review of Research in Open and Distributed Learning, 16(3), 41-64. <https://doi.org/10.19173/irrodl.v16i3.1975>

Driscoll, T. (2012). Flipped Learning and democratic Education. Virginia: The Complete Report. Recuperado de <https://bit.ly/2OXhM88>.

El Miedany, Y. (2019). Flipped learning. En C. Reidsema, L. Kavanagh, R. HadGraft y N. Smith (eds.). The flipped classroom: Practice and practices in higher education (pp. 285-303). Nueva York: Springer. <https://doi.org/10.1007/978-3-319-98213-7_15>

Flores, Ò.; Arco, I. del y Silva, P. (2016). The flipped classroom model at the university: Analysis based on professors' and students' assessment in the educational field. International Journal of Educational Technology in Higher Education, 13(21), 1-12. <https://doi.org/10.1186/s41239-016-0022-1>

Froehlich, D.E. (2018). Non-technological learning environments in a technological world: Flipping comes to the aid. Journal of New Approaches in Educational Research, 7(2), 94-99. <https://doi.org/10.7821/naer.2018.7.304>

Gamboa, Y.G. y SiERra, M.M. (2017). El blog como material de apoyo a la docencia: Estudio de caso en la asignatura de Consulta 1. Biblioteca Universitaria, 20(2), 108-120. <https://doi.org/10.22201/dgb.0187750xp.2017.2.185>

He, W.; Holton, A.; Farkas, G. y Warschauer, M. (2016). The effects of flipped instruction on out-of-class study time, exam performance, and student perceptions. Learning and Instruction, 45, 61-71. <https://doi.org/10.1016/j.learninstruc.2016.07.001>

Hernández, R.; Fernández, C. y Baptista, M.P. (2014). Metodología de la investigación. Madrid: McGraw Hill.

Hinojo-Lucena, F.J.; López, J.; Fuentes, A.; Trujillo, J.M. y Pozo, S. (2020). Academic Effects of the Use of Flipped Learning in Physical Education. International Journal of Environmental Research and Public Health, 17(1), 1-14. <http://dx.doi.org/10.3390/ijerph17010276>

Hinojo-Lucena, F.J.; Mingorance, Á.C.; Trujillo, J.M.; Aznar, I. y Cáceres, M.P. (2018). Incidence of the flipped classroom in the physical education students' academic performance in university contexts. Sustainability, 10(5), 1-14. <https://doi.org/10.3390/su10051334>

Hwang, G.J.; LaI, C.L. y Wang, S.Y. (2015). Seamless flipped learning: A mobile technology-enhanced flipped classroom with effective learning strategies. Journal of Computers in Education, 2(4), 449-473. <https://doi.org/10.1007/s40692-015-0043-0>

Jensen, J.L.; Kummer, T.A. y Godoy, P. (2015). Improvements from a flipped classroom may simply be the fruits of active learning. CBE-Life Sciences Education, 14(1), 1-12. <https://doi.org/10.1187/cbe.14-08-0129>

KHADRI, H.O. (2016). Flipped learning as a new educational paradigm: An analytical critical study. European Scientific Journal, 12(10), 417-444. <https://doi.org/10.19044/esj.2016.v12n10p417> 
Kwan, C. y Foon, K. (2017). A critical review of flipped classroom challenges in K-12 education: Possible solutions and recommendations for future research. Research and Practice in Technology Enhanced Learning, 12(4), 1-22. <https://doi.org/10.1186/s41039-016-0044-2>

LeE, J.; Lim, C. y Kim, H. (2017). Development of an instructional design model for flipped learning in higher education. Educational Technology Research and Development, 65(2), 427-453. <https://doi.org/10.1007/s11423-016-9502-1>

Llanos, G. y Bravo, J. (2017). Flipped classroom como puente hacia nuevos retos en la educación primaria. Revista Tecnología, Ciencia y Educación, 8, 39-49. Recuperado de <https://cutt.ly/1eUx2G6>.

Long, T.; Cummins, J. y Waugh, M. (2017). Use of the flipped classroom instructional model in higher education: Instructors' perspectives. Journal of Computing in Higher Education, 29(2), 179-200. <https://doi.org/10.1007/s12528-016-9119-8>

López, J.; Pozo, S.; Fuentes, A. y López, J.A. (2019). Creación de contenidos y flipped learning: Un binomio necesario para la educación del nuevo milenio. Revista Española de Pedagogía, 77(274), 535-555. <https://doi.org/10.22550/REP77-3-2019-07>

López, J.; Pozo, S. y Pino, M.J. del (2019). Projection of the Flipped Learning Methodology in the Teaching Staff of Cross-Border Contexts. Journal of New Approaches in Educational Research, 8(2), 184-200. $<$ https://doi.org/10.7821/naer.2019.7.431>

Martín, D.; Sáenz, M.; Santiago, R. y Chocarro, E. (2016). Diseño de un instrumento para evaluación diagnóstica de la competencia digital docente: Formación flipped classroom. Didáctica, Innovación y Multimedia, 33, 1-15. Recuperado de <https://bit.ly/2BlOqby>.

McLaughlin, J.E.; Roth, M.T.; Glatt, D.M.; Gharkholonarehe, N.; Davidson, C. A.; Griffin, L.M. ... y Mumper, R.J. (2014). The flipped classroom: A course redesign to foster learning and engagement in a health professions school. Academic Medicine, 89(2), 236-243. <https://doi.org/10.1097/ACM.0000000000000086>

Mengual, S.; López, J.; Fuentes, A. y Pozo, S. (2020). Modelo estructural de factores extrínsecos influyentes en el flipped learning. Educación XX1, 23(1), 75-101. $<$ https://doi.org/10.5944/educxx1.23840>

Moк, H.N. (2014). Teaching tip: The flipped classroom. Journal of Information Systems Education, 25(1), 7-11. Recuperado de <https:/cutt.ly/0eUx3Mp>.

Nikolopoulou, K.; Akriotou, D. y Gialamas, V. (2019). Early Reading Skills in English as a Foreign Language Via ICT in Greece: Early Childhood Student Teachers' Perceptions. Early Childhood Education Journal, 47(5), 597-606. <https://doi.org/10.1007/s10643-019-00950-8>

Nouri, J. (2016). The flipped classroom: For active, effective and increased learningespecially for low achievers. International Journal of Educational Technology in Higher Education, 13(33), 1-10. <https://doi.org/10.1186/s41239-016-0032-z>

Padilla, A.L.; Gámiz, V.M. y Romero, M.A. (2020). Evolución de la competencia digital docente del profesorado universitario: Incidentes críticos a partir de relatos de vida. Educar, 56(1), 109-127. <https://doi.org/10.5565/rev/educar.1088> 
Pereira, S.; Fillol, J. y Moura, P. (2019). El aprendizaje de los jóvenes con medios digitales fuera de la escuela: De lo informal a lo formal. Comunicar: Revista Cientifica Iberoamericana de Comunicación y Educación, 27(58), 41-50. <https://doi.org/10.3916/C58-2019-04>

PÉrez, A. (2018). Uso de smartphones y redes sociales en alumnos/as de educación primaria. Prisma Social: Revista de Investigación Social, 20, 76-91. Recuperado de <https://cutt.ly/reUx7Uw>.

Pozo, S.; López, J.; Moreno, A.J. y López, J.A. (2019). Impact of Educational Stage in the Application of Flipped Learning: A Contrasting Analysis with Traditional Teaching. Sustainability, 11(21), 1-15. <https://doi.org/10.3390/su11215968>

Prieto, A. (2019). Registro de docentes que usan aula invertida (flippers) en España y otros países de habla española. España: docs.google. Recuperado el 10 de noviembre de 2019, de <https://cutt.ly/ZeUz5yy>.

Ribosa, J. (2020). El docente socioconstructivista: Un héroe sin capa. Educar, 56(1), 77-90. <https://doi.org/10.5565/rev/educar.1072>

RuÉ, J. (2007). Enseñar en la Universidad: El EEES como reto para la Educación Superior. Madrid: Narcea.

SAlas, R.A. y Lugo, J.L. (2019). Impacto del aula invertida durante el proceso educativo superior sobre las derivadas considerando la ciencia de datos y el aprendizaje automático. EDMETIC: Revista de Educación Mediática y TIC, 8(1), 147-170. <https://doi.org/10.21071/edmetic.v8i1.9542>

SÁnchez, E.; SÁnchez, J. y Ruiz, J. (2019). Percepción del alumnado universitario respecto al modelo pedagógico de clase invertida. Magis: Revista Internacional de Investigación en Educación, 11(23), 151-168. <https://doi.org/10.11144/Javeriana.m11-23.paur>

Santiago, R. y Bergmann, J. (2018). Aprender al revés. Barcelona: Paidós Educación.

Schmidt, S.M. y Ralph, D.L. (2016). The Flipped Classroom: A Twist on Teaching. Contemporary Issues in Education Research, 9(1), 1-6. <https://doi.org/10.19030/cier.v9i1.9544>

SeERY, M.K. (2015). Flipped learning in higher education chemistry: Emerging trends and potential directions. Chemistry Education Research and Practice, 16(4), 758-768. <https://doi.org/10.1039/c5rp00136f>

SHIH, W.L. y Tsai, C.Y. (2017). Students' perception of a flipped classroom approach to facilitating online project-based learning in marketing research courses. Australasian Journal of Educational Technology, 33(5), 32-49. <https://doi.org/10.14742/ajet.2884>

Souto, A.; Estévez, I.; Iglesias, V. y GonzÁlez, M. (2020). Entre lo formal y lo no formal: Un análisis desde la formación permanente del profesorado. Educar, 56(1), 91-107. <https://doi.org/10.5565/rev/educar.1095>

Tourón, J. y Santiago, R. (2015). El modelo Flipped learning y el desarrollo del talento en la escuela. Revista de Educación, 368, 196-231. <https://doi.org/10.4438/1988-592X-RE-2015-368-288>

TsE, W.S.; ChOI, L.Y. y TANG, W.S. (2019). Effects of video based flipped class instruction on subject reading motivation. British Journal of Educational Technology, 50(1), 385-398.

<https://doi.org/10.1111/bjet.12569> 
TuCKER, B. (2012). The flipped classroom: Online instruction at home frees class time for learning. Education Next, 12(1), 82-83. Recuperado de <https://cutt.ly/ NeUce64>.

Zainuddin, Z.; Habiburrahim, H.; Muluk, S. y Keumala, C.M. (2019). How do students become self-directed learners in the EFL flipped-class pedagogy?: A study in higher education. Indonesian Journal of Applied Linguistics, 8(3). $<$ https://doi.org/10.17509/ijal.v8i3.15270>

Zainuddin, Z. y HaliLi, S.H. (2016). Flipped classroom research and trends from different fields of study. The International Review of Research in Open and Distributed Learning, 17(3), 313-340.

$<$ https://doi.org/10.19173/irrodl.v17i3.2274> 